$\begin{array}{cl}\begin{array}{c}\text { Revue } \\ \text { de } / \text { histoire }\end{array} & \text { Revue de l'histoire des religions } \\ \text { des religions } & \begin{array}{l}1 \mid 2007 \\ \text { Varia }\end{array}\end{array}$

\title{
Rodney Stark et la sociologie religieuse américaine
}

Une stimulation pour la recherche historique européenne

Rodney Stark and the American religious sociology, A stimulation for the

European history research

\section{Frédéric Schwindt}

\section{OpenEdition \\ Journals}

Édition électronique

URL : http://journals.openedition.org/rhr/5235

DOI : $10.4000 /$ rhr.5235

ISSN : $2105-2573$

Éditeur

Armand Colin

Édition imprimée

Date de publication : 1 janvier 2007

Pagination : 61-81

ISBN : 978-2200-92332-7

ISSN : 0035-1423

Référence électronique

Frédéric Schwindt, «Rodney Stark et la sociologie religieuse américaine », Revue de l'histoire des religions [En ligne], 1 | 2007, mis en ligne le 01 mars 2010, consulté le 19 avril 2019. URL : http:// journals.openedition.org/rhr/5235; DOI : 10.4000/rhr.5235 


\section{Rodney Stark et la sociologie religieuse américaine Une stimulation pour la recherche historique européenne}

Après avoir vanté la sécularisation irrémédiable des sociétés modernes, les médias ne parlent plus aujourd'hui que du retour de Dieu. L'école incarnée par le sociologue américain Rodney Stark analyse différemment la situation. Dans la lignée de Max Weber, il est un des rares scientifiques à tenter encore de vastes synthèses du phénomène religieux. Il est aussi l'initiateur de travaux empiriques comme celui où il a montré que les ÉtatsUnis n'étaient pas, au départ, une société très religieuse. Peu connu en France, au contraire du monde anglo-saxon ou de l'Italie, Rodney Stark doit en partie cette désaffection à sa critique de la théorie de la sécularisation, mais aussi à l'utilisation de concepts comme le choix rationnel, la notion de tension ou l'économie de l'offre.

\section{Rodney Stark and the American religious sociology, A stimulation for the European history research.}

Medias used to praise the secularization of modern societies. Now they only deal with the coming back of God. The school which is embodied by American sociologist Rodney Stark analyses the situation in a different way. He follows the lead of Max Weber, and he is the one of the few scientists who dare analyze the religious topic. He is also the man who began important works, such as highlighting that the United States society was not born religious. Unlike English-speaking or Italian people, the French have barely heard about Rodney Stark. The rejection is partly due to criticism of the secularization theory. On the other hand, it is also linked with his using concepts like rational choices, tension and the offer economy. 
La réélection de George W. Bush, le développement des courants évangéliques à l'échelle mondiale, les succès d'éditions d'ouvrages sur Billy Graham ou la Bible Belt (voir la traduction de Au pays de Dieu de Douglas Kennedy ${ }^{1}$ ), mais aussi en France la question du culte musulman, du voile, de la réforme de la loi de 1905 (après celle jadis avortée de la loi Falloux) accompagnent le retour de Dieu comme thème politique, littéraire ou journalistique. Le sujet tend à devenir un véritable marronnier. En nous limitant seulement à l'hexagone, pas un jour en effet sans que ne soit annoncé un dossier dans un mensuel. Les auteurs les plus en vue, les académiciens, Jean-Marie Rouart ${ }^{2}$ en premier lieu, donnent le ton. En trois ou quatre ans, plus d'une dizaine de revues ont été lancées, la plupart pour le grand public. À l'exemple du journal Le Monde, avec Le Monde des Religions, certains quotidiens n'hésitent plus à publier une version dérivée. Il y a quelques années, leurs dirigeants auraient eu peur de prendre un tel risque nancier, mais aujourd'hui l'opération est devenue très pro table. En $\mathrm{n}$, les hommes politiques commencent à se mêler à la discussion suite à l'exemple donné par Nicolas Sarkozy $^{3}$. Ce début du XxI ${ }^{\mathrm{e}}$ siècle connaîtrait-il, comme la $\mathrm{n}$ du $\mathrm{XIX}^{\mathrm{e}}$, une crise de conscience qui avait vu Paul Claudel, derrière son pilier de Notre-Dame, et toute une génération se convertir, de Péguy à Ernest Psichari et de Joris-Karl Huysmans ou Maritain à Jacques Rivière? Régis Debray ne théorise plus le Foco, le foyer d'insurrection guevariste, mais il accumule les analyses érudites du fait religieux ${ }^{4}$. Mieux, dans la préface d'un de ses ouvrages, le romancier, historien et ancien ministre Max Gallo raconte son retour à la foi à l'occasion

1. Douglas Kennedy, Au Pays de Dieu, Paris, Belfond, 2004.

2. Jean-Marie Rouart, Je crois parfois la nuit: Le Libertin chrétien, Desclée de Brouwer, 2004.

3. Nicolas Sarkozy, Thibaud Collin \& Philippe Verdin, La République, les religions, l'espérance, Paris, Pocket, 2005.

4. Plus d'une dizaine d'ouvrages sur ce thème pour le président d'honneur de l'Institut européen en sciences des religions (Ecole Pratique des Hautes Études à Paris) dont: Régis Debray, Dieu, un itinéraire, Paris, Odile Jacob, 2001 ; L'enseignement du fait religieux dans l'école laïque, Paris, Odile Jacob, 2002 ; Le Feu Sacré : Fonctions du Religieux, Paris, Gallimard, 2005. 
du baptême du 1 s du précédent ${ }^{5}$. On se croirait revenu à la vague de la $\mathrm{n}$ du XIX ${ }^{\mathrm{e}}$ siècle mentionnée ci-dessus ${ }^{6}$. On s'interroge, on se félicite ou on s'inquiète sans toujours se rendre compte que ce « revival» avait été annoncé par certains scienti ques alors même que la plupart des spécialistes glosaient sur la « sécularisation » des sociétés modernes. La mort de Dieu avait peut-être été trop vite présentée comme acquise ${ }^{7}$. En tout état de cause, le sujet est aujourd'hui âprement disputé.

Le sociologue Alan Wolfe (Boisi Center for Religion and American Public Life du Boston College) nous décrit une Amérique modérée et en voie de sécularisation, bien loin de l'image renvoyée par les médias ou la personne du président des États-Unis ${ }^{8}$. Il insiste notamment sur les progrès de l'incroyance ou sur le fait que près des deux tiers des Américains sont incapables de réciter cinq des dix commandements. Il est en effet aujourd'hui possible, aux États-Unis, d'assumer publiquement son incroyance (peut-être parce que cela engendre une « tension » valorisante entre l'incroyant et la société ambiante). Même dans les milieux conservateurs, la religion est du reste de plus en plus conçue sur un mode individuel, une sorte de thérapie ou un outil de réalisation personnelle.

Tout aussi critique que lui, Rodney Stark présente une vision complètement inverse, rétive à l'idée de sécularisation et qui insiste

5. Max Gallo, Les Chrétiens (Tome I : Martin, le manteau du soldat), Paris, Fayard, 2002, p. 11-22.

6. Comparaison des deux vagues dans : Frédéric Schwindt, « De Claudel à Max Gallo : le retour de Dieu ? ", Le Géant-Revue du Centre Charles de Gaulle de Nancy, № 2/2004, p. 5-6.

7. Voir à ce sujet l'évolution du sociologue américain Peter L. Berger : Peter L. Berger, La religion dans la conscience moderne - Essai d'analyse culturelle, Paris, Éditions du Centurion, 1971. Peter L. Berger (Ed.), Le réenchantement $d u$ monde, Paris, Bayard, 2001. On remarque que depuis une dizaine d'années, l'homme, défunt en 1968, revient également au centre des préoccupations des philosophes.

8. Alan Wolfe, The transformation of American Religion: How We Actually Live Our Faith, Chicago, University of Chicago Press, 2005. Sébastien Fath présente en revanche une vision beaucoup plus contrastée du président américain et de son entourage : Sébastien Fath, Dieu bénisse l'Amérique - La Religion de la Maison-Blanche, Paris, Seuil, 2004. 
sur le temps long 9 . Au cours des ans, il est devenu l'un des plus importants auteurs anglo-saxons en sociologie et en histoire comparée des religions, ainsi que le chef de le d'une importante école. Il y a peu, il revenait sur une carrière de plus de quarante ans en faisant le bilan de ses recherches dans Exploring the Religious Life ${ }^{10}$.

Abondement cité ou combattu au Québec, aux Pays-Bas, en Belgique ou en Suisse - il vient de publier un livre avec Massimo Introvigne en Italie ${ }^{11}$ - il demeure pourtant largement ignoré en France (même si le dernier rapport parlementaire sur les sectes mentionne ses travaux). Cette méconnaissance sera peut-être palliée par la traduction prochaine d'un de ses ouvrages en français ${ }^{12}$. La répugnance de la recherche française à son endroit provient surtout de ce que Rodney Stark est le vulgarisateur de deux importantes théories venues des sciences économiques et appliquées par lui et ses élèves à l'étude de la religion. Il utilise en effet largement la théorie du choix rationnel et de l'économie de l'offre, alors que l'Europe et la France demeurent attachées au dogme de la sécularisation irrémédiable des sociétés modernes, mariées à l'irrationalité des comportements religieux.

\section{RODNEy STARK}

Né en 1931, ancien journaliste et correspondant de guerre, chercheur à Berkeley où il soutint sa thèse en 1971, Rodney Stark fut professeur de sociologie et de religions comparées à l'université de Washington à Seattle jusqu'en 2004. Il a depuis accepté un poste

9. Rodney Stark, « Secularization: The Myth of Religious Decline », Fides et Historia 385, 1999, p. 286-307.

10. Rodney Stark, Exploring the Religious Life, Baltimore, Johns Hopkins University Press, 2004.

11. Rodney Stark \& Massimo Introvigne, Dio è Tornato - Indagine sulla rivincita delle religioni in Occidente (God is back: An Inquiry into the Revival of Religions in the West), Casale Montferrato, Piemme, 2003.

12. Rodney Stark, One True God: Historical Consequences of Monotheisms, Princeton, Princeton University Press, 2001 (traduction annoncée à Paris aux Éditions Noesis). 
de professeur de sciences sociales à l'université Baylor du Texas où vient de se créer un très important centre pluridisciplinaire voué à l'étude des religions. Il y retrouve deux de ses élèves, le sociologue Christophe Bader et l'historien Paul Froese (spécialiste des religions dans l'ancien empire soviétique). Président de l'Association pour la sociologie des religions dans les années 1980, puis chef du département des religions à l'Association américaine de sociologie en 19961997, Rodney Stark a obtenu de nombreux prix scienti ques et a été nominé au Pulitzer pour son maître ouvrage sur le développement du christianisme dans l'Empire romain ${ }^{13}$. Il a également fondé 1'Interdisciplinary Journal of Research on Religion ${ }^{14}$. Il est l'auteur de plus de 150 articles et de 28 livres, traduits dans de nombreuses langues, la plupart en collaboration avec ses élèves, en premier lieu avec Roger Finke, son ancien assistant de Seattle ${ }^{15}$. Ses préoccupations vont en alternance vers les travaux empiriques et vers de vastes synthèses. Il a ainsi « inventé » une source tout à fait nouvelle pour étudier la question religieuse aux États-Unis depuis l'époque coloniale ${ }^{16}$ et développé une vieille intuition de Durkheim consistant à rapprocher taux de suicides et foi. Mais Rodnay Stark s'est aussi intéressé au monothéisme et il a cherché à dépasser l'idée de Max Weber sur la liaison religion-capitalisme en étendant le propos à l'idée de progrès et aux raisons du succès de l'Occident ${ }^{17}$. Il est en effet l'un des rares aujourd'hui à tenter de dépasser les enquêtes fragmentaires sur tel ou tel culte, telle ou telle époque et tel ou tel espace géographique au pro $t$ d'une vue globale du phénomène religieux ${ }^{18}$.

13. Rodney Stark, The Rise of Christianity: A sociologist reconsiders History, Princeton, Princeton University Press, 2001.

14. Version numérique accessible sur : http://www.religjournal.com

15. Aujourd'hui professeur de sociologie à l'université d'Etat de Pennsylvanie. Pour une bibliographie complète de Rodney Stark, voir le site de l'université Baylor : www.baylor.edu/sociology

16. Rodney Stark, "A Note on the Reliability of Historical U.S. Census Data on Religion », Sociological Analysis 52, 1992, p. 91-95.

17. Rodney Stark, The Victory of Reason: How Christianity Led to Freedom, Capitalism, And Western Success, Random House, 2005.

18. Rodney Stark \& Roger Finke, Act of Faith - Explaining the human face of Religion, Berkeley - Los Angeles - London, University of California Press, 2000. 


\section{L'ANCIEN PARADIGME : LE DOGME DE LA SÉCULARISATION}

Religion et modernité ne feraient pas bon ménage. Dans un ouvrage récent, Rodney Stark a largement contredit cette af rmation ${ }^{19}$. En effet, le sociologue aime prendre les problèmes à rebours et casser les certitudes. Il y a quelques années, il a ainsi conduit, avec d'autres, une étude sur les convictions religieuses des universitaires américains en partant du présupposé classique que les spécialistes des sciences dures devraient être les plus détachés de la foi, en tout cas les plus sceptiques. Quelle ne fut pas sa surprise de découvrir chez les mathématiciens et les physiciens le plus fort taux d'adhésion (plus que chez certains membres des départements de théologie) et que ce taux décroissait régulièrement vers les spécialités de sciences humaines ou de littérature ${ }^{20}$ ! Donc l'opposition entre science et foi n'est peut-être pas aussi tranchée que cela.

Encore faudrait-il examiner les concepts qui ont servi de fondements à cette théorie. Le progrès technique, l'élévation du niveau de vie, le passage des sociétés holistes aux sociétés individualistes, le développement de la démocratie et du libéralisme entraineraient irrémédiablement un déclin des religions (il faudrait d'ailleurs distinguer religions et sentiment religieux, ce qui n'est pas exactement la même chose). Or, ceci n'est pas une démonstration scienti que mais la simple généralisation de l'expérience vécue par l'Europe depuis les Lumières puis la Révolution industrielle (quoique les historiens remettent aujourd'hui en cause l'idée d'une adhésion unanime des habitants sous l'Ancien Régime). Mais la sécularisation est maintenant présentée comme un dogme, une évolution obligatoire et inévitable, un modèle universel. Dans la ligne droite de Voltaire, le propos historique ou sociologique est fréquemment contaminé par des jugements de valeur : la sécularisation serait liée au progrès et au bien, la religion à l'obscurantisme supposé des siècles passés. N'était-ce pas l'opium du peuple?

La ré e xion sur ces problèmes est biaisée par des considérations politiques, que ce soit aux États-Unis par la montée en puissance de

19. Rodney Stark, The Victory of Reason, op. cit.

20. Rodney Stark \& Roger Finke, Act of Faith, op. cit., p. 52-53. 
la droite fondamentaliste, observée depuis les années 1980, ou en France par la question de la laïcité. Pour prendre un exemple dans l'actualité, a n de montrer que ce débat ne concerne pas que les spécialistes, la question de l'adhésion de la Turquie à l'Union Européenne comporte en elle-même une croyance liée à la théorie de la sécularisation. La croissance économique qui suivrait cette adhésion, le développement des classes moyennes et le progrès de la démocratie permettraient d'y réduire l'in uence sociale de l'Islam. On ne fait hélas pas attention au fait que, depuis vingt ans, le taux de croissance moyen de la Turquie est le double de celui de l'Europe de l'Ouest et que cela n'a en rien freiné le développement du fondamentalisme. Pour la Turquie, ce raisonnement sous-estime l'impact de groupes religieux d'origine chiite, comme les Alévis, qui sont des facteurs de stabilité et de progrès (par la valorisation du statut de la femme, de l'éducation ou de la liberté individuelle). Il y a donc là le risque d'un raisonnement circulaire : si les choses se sont déroulées apparemment comme cela, c'est qu'elles ne pouvaient que se passer comme cela. D'où des annonces prophétiques, il y a une trentaine d'années, qui promettaient la disparition des religions, d'abord en Europe puis aux États-Unis avant le début du XXI ${ }^{\text {e }}$ siècle.

Or, les travaux les plus récents montrent le retour des croyances même au sein de la vieille Europe. Rodney Stark et Massimo Introvigne peuvent même parler d'un « revival » italien à l'œuvre depuis le début des années $1980^{21}$. La croyance en l'existence de l'âme a augmenté de $10 \%$, celle en la vie après la mort ou aux enfers de $15 \%$ ( $25 \%$ pour les seuls $18-29$ ans). Chez les jeunes, l'idée que Dieu n'est pas important dans la vie est même passée de $19 \%$ en 1981 à seulement $8 \%$ en 1999. On note également un retour en grâce de la prière. L'actualité tend donc à détruire le caractère inéluctable de la sécularisation.

En y pensant bien, la théorie de la sécularisation liée à l'idée de progrès est éminemment religieuse. Monisme axiologique, elle conduit en ligne droite d'un point $\mathrm{A}$ à un point $\mathrm{B}$ xé d'a vance. Que

21. Massimo Introvigne \& Rodney Stark, «Religious Competition and Revival in Italy: Exploring European Exceptionalism », Interdisciplary Journal of Research on Religion, volume 1, 2005, Article 5. 
l'on appelle cela bonheur, société parfaite, parousie et jugement dernier ou même $\mathrm{n}$ de l'Histoire dans la perspecti ve d'Alberto Fukuyama (soit l'établissement général de la démocratie et de la société libérale) ${ }^{22}$, voire même Révolution (Marx reprend le même schéma linéaire), les différences sont faibles. Auguste Comte, dans sa théorie des trois états, posait déjà les bases d'un tel schéma : état magique, état théologique et état positif. N'oublions cependant pas qu'il affectait une " race » à chaque étape et bien sûr que seule la race blanche atteignait le stade scienti que dans son modèle. Il n'est donc pas inutile d'examiner l'origine et les fondements des concepts que nous utilisons couramment pour débattre, parfois sans $\mathrm{y}$ faire attention. C'est d'ailleurs le piège dans lequel tombent de nombreux historiens qui reprennent à leur compte des notions issues des sciences sociales (pas toujours en l'avouant d'ailleurs dans leurs notes) sans s'interroger sur le contexte dans lequel ils ont été conçus.

Dans la lignée de la théorie de la sécularisation, la crise de l'Église catholique, la chute de la pratique avec celle des ordinations dans le monde occidental sont généralement analysées comme la conséquence d'une inadéquation à la société moderne. La seule réponse possible consisterait en une série d'aggiornamento remettant en cause le gouvernement monarchique de l'Église, sa structure centralisée, son dogme, sa morale, etc. Or, depuis Vatican II, l'in uence du catholicisme continue de diminuer en Europe. Les pays du Sud occupent un poids de plus en plus important, en Amérique latine notamment. Mais même là, le catholicisme est de plus en plus concurrencé par les Églises évangéliques ou les cultes syncrétiques. Or, il apparaît que ces religions nouvelles se développent parce qu'elles remplissent des fonctions que le catholicisme a abandonnées, notamment dans le domaine de l'organisation de la communauté d'habitant ou de la circulation des ressources ${ }^{23}$. Rodney Stark avance donc l'idée hérétique que le Concile et la volonté d'adapter

22. Alberto Fukuyama, End of History and the Last Man, Free Press, 2006 (dernière édition en date).

23. Jean-Pierre Bastian, Protestantisme en Amérique latine - Une approche socio-économique, Genève, Labor et Fides, 1994 ; " Amérique latine : la vague pentecôtiste », Sciences humaines Hors série N 41/juin-août 2003, p. 42-44. 
le catholicisme au siècle n'ont pas freiné le mouvement, mais l'ont au contraire accéléré en réduisant la tension (nous reviendrons plus tard sur cette idée) entre l'Église et le monde.

\section{UNE THÉORIE GLOBALE DU RELIGIEUX}

Dans Act of Faith - Explaining the Human Side of Religion, paru en 2000, Rodney Stark et Roger Finke reviennent sur trente ans de travaux empiriques et d'études de terrain a $\mathrm{n}$ de fournir un modèle en 32 dé nitions et 99 propositions. Pour eux, la religion s'e xplique d'abord et en priorité au niveau de l'individu. Ils s'attachent donc à décrire les micro-fondements de la religion, les choix effectués par les dèles (autour des notions de con version et surtout de réaf liation), ainsi que la dynamique des groupes.

\section{Le choix rationnel}

La recherche partait autrefois (et encore souvent aujourd'hui) du principe que le sentiment religieux est quelque chose de mystérieux, donc d'incompréhensible, et qu'il n'est pas nécessaire, voire impossible, de l'étudier sur le fond. Il appartient au domaine de l'irrationnel et ne relève donc pas de la science qui doit seulement s'intéresser aux structures, aux gestes et aux comportements. À l'inverse, la neurobiologie et les sciences cognitives tendent aujourd'hui à identi er dans le fonctionnement du cerv eau une prédisposition à la religion ${ }^{24}$. Si l'explication se veut rationnelle, elle tend à évacuer le rôle du sujet. En effet, même si elle n'explique que les fondements généraux de la croyance, laissant aux sociétés humaines et à l'histoire culturelle la liberté de faire évoluer les formes de la religion, l'individu reste en quelque sorte victime de son cerveau modelé par des millénaires d'évolution.

24. Pascal Boyer, Religion Explained, New York, 2001 ; « De la Religion aux neurones - D'où viennent le succès de certains concepts religieux ? », $L a$ Recherche Hors Série $\mathrm{N}^{\circ} 14$ Janvier-Mars 2004, p. 48-50. 
Rodney Stark et son école s'appuient au contraire sur l'idée que le croyant agit - le citoyen romain, le paysan européen de l'époque moderne, le bourgeois capitaliste du XVIII ${ }^{\mathrm{e}}$ siècle, comme le pasteur baptiste d'aujourd'hui - d'une manière tout à fait raisonnable et que son adhésion, ainsi que ses choix, sont dictés par tout un processus de ré e xion et l'espoir d'un retour. Ceci implique un calcul individuel, personnel a $\mathrm{n}$ de maximiser ce retour par rapport aux efforts nécessités par l'acte de foi (c'est ce qu'on appelle en économie un coût). Bien sûr, ce retour est attendu dans un autre monde, mais pas seulement. Le sociologue de l'université Baylor applique donc à l'étude de la religion la notion de choix rationnel conçue par les sciences économiques ${ }^{25}$.

La théorie selon laquelle les croyances reposent sur des choix rationnels tend aujourd'hui à se répandre chez les sociologues, moins chez les historiens. En France, les travaux de Gérald Bronner ${ }^{26}$ et bien sûr ceux de Raymond Bourdon ${ }^{27}$ vont dans ce sens. Dans Le pouvoir au village, son maître ouvrage où il réalise la biographie d'un exorciste du XVII ${ }^{\mathrm{e}}$ siècle ${ }^{28}$, le spécialiste de la microstoria Giovanni Levi avance l'hypothèse de l'existence d'une rationalité restreinte. Les philosophes et les sociologues sont eux aussi de plus en plus nombreux à parler de cette rationalité limitée ou subjective. Selon l'historien italien, les croyances et l'irrationalité apparente seraient un moyen de se projeter dans le futur et de plani er un a venir incertain, du moins dans l'environnement mental qui est celui des habitants. Or, il s'agit presque exactement de la dé nition donnée par Rodne y Stark et Roger Finke : "Within the limits of their information and understanding, restricted by avaible options, guided by their preferences

25. Laurence R. Iannacone, Rodney Stark \& Roger Finke, « Rationality and the Religious Mind », Economic Inquiry 36, 1998, p. 189-214.

26. Gérald Bronner est maître de conférence en sociologie à l'université de Nancy II et il cherche à expliquer le maintien des croyances dans nos sociétés dites modernes : Gérald Bronner, L'Empire des croyances, Paris, P.U.F., 2003.

27. Raymond Bourdon, "Toward a Synthetic Theory of Rationality », International Studies of Philosophy of Science $\mathrm{N}^{\circ}$ 7, 1993, p. 5-19; Raison, bonnes raisons, Paris, P.U.F., 2003.

28. Giovanni Levi, Le pouvoir au village - Histoire d'un exorciste dans le Piémont du XVII siècle, Paris, N.R.F.-Gallimard, 1989 (« Bibliothèque des Histoires »). 
and tastes, humans attempt to make rational choices. ${ }^{29 "}$ "Nous-même avons remarqué que les croyances et les diverses pratiques proposées par la religion permettaient de maintenir l'unité du groupe et de mobiliser la communauté d'habitants vers des actions collectives destinées à assurer la survie, tout en permettant à chaque individu d'y trouver son compte ${ }^{30}$.

\section{La notion de tension}

Les conceptions de Rodney Stark et de son école reposent également sur la notion de «tension » qui a largement occupé le sociologue au cours des années 1960 et $1970^{31}$. Un groupe religieux progresse lorsque son mode de vie et les choix moraux qu'il enjoint à ses dèles s'écartent de la norme acceptée par la société du temps. Sinon, on ne peut expliquer, après 1776, le lent déclin de la Main Line, le groupe de congrégations protestantes (épiscopaliens, congrégationalistes, presbytériens...) qui dominait la société coloniale nord-américaine, au pro $\mathrm{t}$ de confessions nouv elles, alors moins politiquement correctes dans leurs pratiques. Les Églises établies critiquent en effet fortement la pastorale des méthodistes et des baptistes, leurs pasteurs non-professionnels ou leurs prédicateurs itinérants, les célèbres circuit-riders dont Francis Ashbury (17451816) fut un des modèles. Sont donc visés pêle-mêle les tournées de prédication, les camps d'été où les dèles se retrouv ent, ainsi que le système des free churches. Les sermons prêchés sur un mode dynamique et passionnel par des pasteurs non gradués en théologie apparaissent surtout incorrects et scandaleux au clergé traditionnel de la côte Est.

Or, lorsque ces nouvelles congrégations se stabilisent et adoptent le mode de fonctionnement des premières (méthodistes et baptistes du Nord à partir de 1850), elles entament leur propre décadence.

29. Rodney Stark \& Roger Finke, Act of Faith, op. cit., p. 277.

30. Frédéric Schwindt, La communauté et la foi - Confréries et société dans l'ouest de l'espace lorrain (XIII ${ }^{e}$ XX ${ }^{e}$ siècles), Thèse d'Histoire de l'Université de Nancy II sous la direction du professeur Louis Châtellier, 2004.

31. W.S. Bainbridge \& Rodney Stark, "Sectarian tension », Review of Religious Research 22, 1980, p. 105-124. 
Rodney Stark démontre ainsi, mais il ne fait en cela que suivre l'enseignement de Max Weber, que la secte des témoins de Jéhovah est entrée sur la voie de l'assimilation car une partie d'elle-même commence à transiger avec le monde, en acceptant par exemple la transfusion sanguine. À mesure qu'elle passe du rang de secte (au sens weberien) à celui d'Église, elle perd par contrecoup une part de son pouvoir d'attraction.

Tout l'intérêt de la notion de tension est qu'elle donne du poids au choix rationnel du croyant en accordant un prix à l'engagement. Choisir telle ou telle confession, mais aussi en changer n'est pas anodin en termes de coût personnel et de capital social (autre notion importante trop ignorée des historiens). Un vieux proverbe communiste, transférable ici, ne disait-il pas que plus on donne de travail à un militant et plus cela lui coûte d'efforts, d'abnégation et d'épreuves (prisons, coups, regard des autres et rejet de la part de la société), plus l'individu demeure attaché au Parti et aux liens qui le relient aux autres adhérents. Les nouveaux convertis iraient donc en priorité vers des cultes à la forte visibilité sociale, ceux dont les exigences sont suf samment fortes pour les mettre en situation de rupture a vec la société ambiante, voire avec leurs propres familles. Ainsi, on note depuis vingt ans une augmentation régulière des conversions d'Américains au judaïsme. Les raisons en sont multiples, mais les efforts demandés ne sont pas sans lien avec l'intérêt de la conversion.

Notre regard s'est peut-être donc trop attardé sur la religion à la carte et les cultes peu exigeants qui facilitent le zapping et le turn over des dèles, un mot qui d'ailleurs n'a plus de sens au sein de ces organisations. Ceux-ci auraient caché les vrais mouvements de fond dont on voit seulement maintenant émerger quelques parties, en particulier la face fondamentaliste à rebours de la prétendue sécularisation. Toute une relecture de l'histoire des Mormons, dont Rodney Stark est aussi l'un des spécialistes ${ }^{32}$, est en train d'être faite à partir de la notion de tension. On pourrait également appliquer le même raisonnement à l'Islam européen et aux nouveaux musulmans,

32. Rodney Stark \& Reid L. Neilson (Ed.), The Rise of Mormonism, New York, Columbia University Press, 2001. 
non pas issus de l'immigration, mais des familles de souche. Ceuxci se montrent en effet souvent « plus royalistes que le roi ».

C'est donc moins dans le contexte, même si les tenants de la théorie du marché religieux ne l'évacuent jamais, que dans l'économie interne des mouvements qu'il faut chercher les clefs de leurs succès ou de leur déclin.

\section{UN GRAND LIVRE D'HISTOIRE ÉCRIT PAR DEUX SOCIOLOGUES : The Churching of America - 1776-2005}

Le plus grand apport du maître ouvrage que Rodney Stark et son élève Roger Finke ont consacré à l'histoire des Églises américaines depuis $1776^{33}$, est de casser le double mythe du May Flowers et de la Petite maison dans la prairie. Contrairement aux idées reçues, l'Amérique des Pères fondateurs n'était pas une nouvelle Jérusalem constituée de puritains soucieux de construire la cité de Dieu sur terre. Cette vision est une reconstruction a posteriori à laquelle les historiens américains classiques ont donné crédit. Au moment de la guerre d'Indépendance, seulement $17 \%$ des habitants des ÉtatsUnis reconnaissaient appartenir à une Église et ce taux, qui a peiné à monter à un tiers de la population après 1800 , demeura stable malgré l'immigration dans la deuxième moitié du XIX siècle. La critique rétorquera que croire et adhérer à une Église sont deux choses différentes. C'est pourtant le même argument que celui que l'on oppose aujourd'hui, en Europe, aux partisans de la sécularisation lorsque l'on compare le déclin des cultes organisés, celui des Églises of cielles, et le maintien de certaines cro yances révélé par les études d'opinion. Tardivement, à partir de 1890, l'Amérique aborde une nouvelle étape de son histoire religieuse avec la croissance régulière et durable de son taux d'encadrement des dèles, d'où le titre du li vre

33. Rodney Stark \& Roger Finke, The churching of America - 1776-2005 - Winners and losers in our religious economy, New Brunswick - New Jersey, Rutgers University Press, 2005 (version augmentée et actualisée de la première édition de 1992). 
des deux sociologues et l'utilisation du mot churching ${ }^{34}$. Ce taux passe de $41 \%$ en 1906 à $59 \%$ en 1952 et presque $70 \%$ aujourd'hui avec le phénomène très médiatisé des born again (en raison notamment de la personne du président des États-Unis) qui constitueraient près du tiers des dèles américains. L'analyse donnée par les deux sociologues a pour nous le grand avantage de replacer les réalités actuelles au sein d'une longue histoire et de montrer sur quoi elles s'appuient.

Les auteurs prouvent surtout que les réussites ou les échecs des différentes Églises ne sont pas seulement le produit de l'immigration, beaucoup de nouveaux Américains étant au départ détachés de la religion ou bien rompant avec leurs origines. Plus du tiers des immigrants catholiques d'origine européenne ne demeurent ainsi pas dèles à leur culte maternel. Stark et Fink e, à la suite d'Andrew Greeley, montrent également que nombre d'Irlandais venus en Amérique n'étaient pas des catholiques, mais des protestants émigrés des comtés d'Hulster ${ }^{35}$. D'ailleurs, les deux phénomènes s'additionnant, la majorité des descendants d'Irlandais (mais aussi de Français) qui vivent aujourd'hui aux États-Unis sont protestants. Cette approche s'oppose donc aux idées toutes faites sur les causes du succès catholique aux États-Unis car beaucoup de catholiques actuels, notamment dans le Middle West, ne sont pas des dèles de souche de l'obédience romaine mais des descendants de protestants. Au bon moment, dans la seconde moitié du XIX ${ }^{\mathrm{e}}$ siècle, et alors même que la dynamique méthodiste commençait à retomber, l'Église catholique a su faire évoluer son organisation et sa pastorale au contact des mouvements évangéliques en imitant ce qui permettait à ceux-ci de réussir. Ainsi, le père Francis Xavier Wenniger (1805-1888) adopta pratiquement l'existence et les manières de prêcher d'un circuit-rider méthodiste. Active dans la compétition, elle put reprendre à son compte une part appréciable du marché religieux.

34. Jeu de mots sur le sens originel qui signi e «relevailles » et l'idée d'encadrement croissant de l'Amérique par ses Églises.

35. Andrew Greeley, «Ethnic Variations in Religious Commitment» in Robert Wuthnow (Ed.), The Religious dimension: New Directions in Quantitative Research, New York, Academic Press, 1979. 
Pour ce qui est des cultes protestants, il est intéressant de noter que les Églises qui se développèrent le plus sont à la fois celles pour lesquelles la rigidité du dogme est importante et celles chez qui demeure l'image d'un Dieu fort. Nous sommes loin des Unitariens qui n'obligent pas même à croire en Dieu. Elles sont les moins centralisées et elles détiennent le clergé le moins bien formé (souvent rien d'autre que l'école primaire) et le moins bien payé (d'où l'obligation d'avoir un emploi). Moins une Église est instituée, plus elle réussit. Et lorsque l'une d'entre-elles négocie avec le siècle, décide d'élever le niveau d'éducation de ses pasteurs, notamment en théologie, en créant lycées, séminaires, et universités, de construire des chapelles $\mathrm{x}$ es et de les fédérer, elle commence à perdre de l'in uence. Ainsi s'expliquent, à la $\mathrm{n} \mathrm{du} \mathrm{XVIII}^{\mathrm{e}}$ ou dans la première moitié du XIX ${ }^{\mathrm{e}}$ siècle, les progrès du Méthodisme aux dépens de la Main Line, le groupe d'Églises of cielles, puis ensuite la montée en puissance des Baptistes. Ceux du Sud progressent beaucoup plus que ceux du Nord, qui ont adopté le mode de fonctionnement dominant et lorsque les Églises méthodistes renoncent à ce qui avait fait leur succès depuis l'époque du Grand Réveil, ils deviennent la première dénomination religieuse aux États-Unis.

\section{L'ÉCONOMIE RELIGIEUSE ET LA THÉORIE DE L'OFFRE}

Les croyances seraient moins importantes que les organisations, la sociabilité et les phénomènes de groupe qui conduisent les dèles vers certains cultes et non vers d'autres. C'est pourquoi les enquêtes s'orientent de plus en plus vers l'analyse des phénomènes sectaires. Le sociologue Chao Hsing-Kuang a ainsi démontré que les conversions d'immigrés chinois en faveur d'un groupe sectaire protestant de San Francisco étaient guidées par une revue, mais que ce n'était pas les articles de fond, le dogme et les croyances, mais les petites annonces annexées au journal qui servaient de support à la création de réseaux de soutien mutuel préalables au changement religieux ${ }^{36}$.

36. Chao Hsing-Kuang, « The Converts in Taiwanese Immigrant Church », Soochow Journal of Sociology 9, March 2000. 
Au début du $\mathrm{XX}^{\mathrm{e}}$ siècle, le diocèse de Verdun, dans l'Est de la France, tente une relance organisée de la sorte ${ }^{37}$. Un journal mensuel, l'Etincelle, tiré à 20000 exemplaires, sert à faire circuler l'information et à créer des liens entre 360 associations du Sacré-Cœur réparties dans les 550 paroisses. Les confréries regroupent environ 30000 membres, ce qui est énorme pour un département de moins de 300000 âmes. L'élite du mouvement est formée de zélatrices qui, comme les Témoins de Jéhovah, livrent la revue aux membres et font la tournée du village pour trouver de nouveaux membres. Le journal donne en exemple les meilleurs prosélytes et propose un service de petites annonces qui permet d'assurer une multitude de services. Par ce moyen, l'Église catholique, loin d'être victime de la loi de Séparation, passe à l'offensive grâce à une stratégie qui lui apporte un sérieux atout « commercial».

Rodney Stark et ses collègues appliquent donc à la religion le concept d'économie religieuse parfois décliné sur le mode de la théorie de l'offre. C'est une théorie issue directement des sciences économiques, qui s'appuie tout naturellement sur les notions de choix rationnel et de coût présentées plus haut, mais aussi de compétition. Dans cette démarche, le sociologue de l'université Baylor a retrouvé l'économiste californien Laurence R. Iannacone, précurseur des recherches dans ce domaine ${ }^{38}$. Appeler une théorie « le marché de la religion » a suscité de nombreuses critiques, notamment celle de banaliser la foi en s'abaissant à l'étudier à l'aune de la concurrence. Bien sûr, ces critiques sont surtout venues de ceux qui pensaient que la religion est un fait irrationnel, un reste d'obscurantisme, voire même le symptôme d'une grave psychopathologie. Bien entendu, l'économie religieuse est bien plus subtile qu'une simple description des pratiques commerciales à l'œuvre au sein des Églises américaines, pratiques qui expliquent l'acceptation of cielle de la

37. Frédéric Schwindt, La communauté et la foi, op. cit. Archives départementales de la Meuse 616 PER.

38. Laurence R. Iannacone, « Religious Participation : A Human Capital Approach », Journal for the Scientific Study of Religion 29(3), September 1990, p. 297-314 ; "Social Markets and the Economic of Religion », Social Compass 39(1), March 1992, p. 121-131. 
Scientologie comme religion par l'Etat américain (avec les avantages scaux af férents). Et puis le vocabulaire employé par Rodney Stark - qui aime provoquer - ne facilite pas toujours l'acceptation de ses idées. Ainsi utilise-t-il le terme de firm pour désigner les Églises en lutte les unes contre les autres pour la captation d'un segment du marché de la religion.

Ce qu'on appelle une économie religieuse, ce sont, au-delà des phénomènes nanciers, les mécanismes matériels à l'œuvre dans le fonctionnement des religions ou à l'origine des conversions et des réaf lations ${ }^{39}$ du fait même de l'existence d'un marché où les cultes se mesurent et se concurrencent. Ce modèle se décline en de nombreuses variantes selon que le marché est libre ou pas, régulé éventuellement par l'Etat ou par une Église of cielle qui pro voque une distorsion de concurrence, etc. Un marché libre et une forte concurrence, la liberté ou non de proposer une offre variée qui s'adapte aux besoins des consommateurs (c'est l'application littérale de la théorie de l'offre), le devoir même de s'adapter à la demande pour survivre expliqueraient un fort taux de pratique religieuse, ou tout au moins, d'adhésion aux Églises. C'est en tout cas grâce à ce modèle que Rodney Stark et Massimo Introvigne expliquent le revival religieux italien récent ${ }^{40}$.

Certains apportent pour preuve de l'inanité de ces théories le cas des États musulmans qui possèdent une religion d'État, donc un marché non libre, et un fort taux de pratique religieuse. Reprenant à notre compte le dogme de la sécularisation, nous pourrions invoquer le fait que ces pays sont inégalement avancés dans le processus historique de détachement. Mais la chute importante de la fécondité dans plusieurs d'entre eux, récemment entrés dans la transition démographique, nous en apprend bien plus sur la perte d'audience

39. Conversion : changement au pro t d'une religion issue d'un environnement socioculturel tout à fait différent de celui de l'individu (par exemple $\mathrm{du}$ catholicisme vers le bouddhisme). Réaf liation : changement au pro $\mathrm{t}$ d'un culte appartenant au même environnement socioculturel que celui de l'individu ou au sein de la même famille religieuse (par exemple d'une Église réformée à une Église évangélique).

40. Massimo Introvigne \& Rodney Stark, «Religious Competition and Revival in Italy », op. cit. 
de la religion of cielle que le discours sur le statut de la femme. Ces États, même l'Arabie saoudite, font aujourd'hui face à une vague de conversion au christianisme qui les conduit à réactiver une législation punissant parfois de mort la « trahison » (Afghanistan). Même l'Algérie est en train de voter des lois pénalisant le prosélytisme et la conversion. Il est vrai que les troubles connus par ce pays et le problème des minorités, notamment la question kabyle, ont conduit le marché religieux à une dérégulation de fait. Depuis 1979, l'Iran des Mollahs lui-même n'est pas épargné par des conversions, certes cachées et peu nombreuses numériquement, mais symboliquement marquantes au pro $t \mathrm{du}$ culte zoroastrien. La Chine Populaire possède également «un marché interdit». Elle voit pourtant une progression sans précédent de l'af liation aux Églises et à une multitude de sectes, justi ant l'invention par un chercheur de l'expression de «marché noir » de la religion ${ }^{41}$.

\section{UNE SOURCE D'INSPIRATION POUR L'HISTOIRE RELIGIEUSE EUROPÉENNE}

La théorie de l'offre apporte une stimulation importante à l'étude de certains phénomènes historiques et pas seulement aux problèmes actuels. Les historiens du vieux continent tireraient d'ailleurs un grand pro $t \mathrm{du}$ rapprochement des concepts, des méthodes et des conclusions de la sociologie américaine actuelle (notamment celles tirées de The Churching of America) avec ce que nous savons de la Réforme catholique des XVII et $\mathrm{XVIII}^{\mathrm{e}}$ siècles ou bien des tentatives de reconquête religieuse du XIX $\mathrm{X}^{\mathrm{e}}$ siècle. En effet, la recherche a partiellement expliqué ces phénomènes par le développement des séminaires et donc d'un clergé séculier mieux formé. Mais la plupart des missionnaires et des simples prêtres de l'époque moderne n'avait pas encore béné cié d'une telle formation. De même, après la Révolution, la France mit plusieurs décennies pour reconstituer son clergé paroissial. Avant que les séminaires ne fonctionnent de nouveau

41. Fenggang Yang, «The Red, Black and Gray Markets of Religion in China ", Paper presented at the Annual Meeting of the Society for Scienti c Study of Religion, Norfolk, Virginia, 2003. 
à plein, elle dut pallier les dif cultés comme elle le pouv ait. Ainsi, Jean-Marie Vianney (1786-1859), le célèbre curé d'Ars qui attire les foules dans sa paroisse des Dombes, est entré dans les Ordres sur le tard parce qu'on jugeait son bagage intellectuel trop léger. Tant au XVIII ${ }^{\mathrm{e}}$ qu'au XIX ${ }^{\mathrm{e}}$ siècle, le début du détachement religieux paraît coïncider avec le moment où les séminaires fournissent des curés à la fois nombreux et au niveau d'éducation élevé. Paradoxe? À l'aune de l'exemple américain, on peut légitimement se demander si l'élévation du niveau de formation des prêtres n'a pas contribué à une rigidi cation du clergé, un élément qui a pu provoquer ou accompagner le retrait des dèles ${ }^{42}$.

Toute une relecture des origines de la Réforme, puis de son éclatement en courants divers, serait à faire autour des notions de choix, de demande et de concurrence. Si l'on n'ignore pas l'angoisse qui était celle des contemporains de Luther, si l'on connaît leur attente d'une religion plus attentive à leurs interrogations ou bien d'un clergé moins corrompu, on a trop insisté sur le rôle des grands réformateurs ou des princes. Sans aller plus avant dans le détail, le livre de raison de Gilles de Gouberville, un petit seigneur normand mort en 1578, nous montre un homme qui hésite entre la messe et le prêche et qui va donc aux deux, le temps de se faire un avis ${ }^{43}$. Certes, il s'agit d'un gentilhomme, mais l'homme du peuple était aussi capable de décider par lui-même.

À l'inverse, on imagine fréquemment la Contre-réforme - la Réforme propre au catholicisme qui trouve sa source dans le Concile de Trente (1545-1563) - comme un phénomène massif, frontal et brutal d'acculturation religieuse, la volonté d'appliquer une norme unique à l'ensemble de la société chrétienne. Face à des populations soumises, au mieux passives, la Réforme tridentine aurait envoyé ses milices constituées par des Ordres religieux et monastiques nouveaux ou rénovés, prêts à obéir, à l'image des Jésuites, perinde ac cadaver. Mais, il n'y a jamais eu en fait de plan coordonné, ni de « complot jésuite ». Cette image est une création de la littérature du XIX ${ }^{\mathrm{e}}$ siècle.

42. Hypothèse que nous défendons dans : Frédéric Schwindt, La communauté et la foi, op. cit.

43. Madeleine Foisil, Le Sire de Gouberville, Paris, Flammarion, 1986. 
En revanche, même un peu anarchique, la oraison religieuse des $\mathrm{XVI}^{\mathrm{e}}$ et $\mathrm{XVII}{ }^{\mathrm{e}}$ siècles, et par bien des aspects encore celle du siècle des Lumières, est assimilable à une « relance par l'offre». La fondation de centaines de couvents, la multiplication des formes de dévotion (Rosaire, Saint-Sacrement, plus tard le Sacré-Cœur...), la réaf $r$ mation du culte des saints, des reliques et des images, la création de milliers de confréries ${ }^{44}$ ont permis une adaptation des décisions du Concile aux réalités locales et aux dèles de réaliser de vrais choix parmi cette offre vaste.

Loin du village immobile, et pas seulement dans le domaine religieux, l'historiographie tend en effet aujourd'hui à réhabiliter les habitants qui ne demeurent pas passifs face à la modernité et à l'intrusion dans leur monde du pouvoir royal ou de la Réforme catholique $^{45}$. La combinaison du choix rationnel et de la théorie de l'offre au sein du modèle de l'économie religieuse permet d'expliquer la complexité d'un mouvement comme la Réforme catholique en insistant sur la rencontre d'une volonté et du terrain. Mieux, la concurrence entre les maisons religieuses ou entre les réguliers et les séculiers, observée à l'échelle urbaine ou à l'occasion des missions, loin d'être la tare dénoncée à l'époque par les visiteurs, est l'une des clefs du système. Du fait de cette compétition, les religieux réalisent de véritables études de marché a n de saisir un segment de la population, donc du marché local, en cherchant un positionnement intéressant, en diversi ant leur of fre ou en passant des accords avec d'autres couvents, parfois pour échanger un quartier, une chapelle ou une dévotion. Selon nous, ceci nous rapproche d'une économie religieuse telle qu'elle a été dé nie par Rodney

44. La bibliographie devient très importante pour ce sujet. Un des grands apports de la recherche récente est constitué par le dépouillement des fonds du secrétariat des brefs réalisé à Rome par Philippe Boutry, Bernard Dompnier et Marie-Hélène Froeschlé-Chopard. Cette étude permet de se faire une idée de la géographie des fondations de confréries à l'échelle de la Chrétienté.

45. Par exemple, le public, loin d'être victime de la justice, sait très bien jouer des tribunaux et effectuer des choix parmi les procédures possibles, voire passer au moment opportun de l'une à l'autre : Hervé Piant, Une justice ordinaire - Justice civile et justice criminelle dans la prévôté royale de Vaucouleurs sous l'Ancien Régime, Rennes, P.U.R., 2006. 
Stark et Laurence R. Iannacone. La concurrence et l'existence d'un marché ont même été une chance pour l'Église catholique, en tout cas l'une des causes de son succès qu'il nous faut à présent étudier puisque l'Histoire continue.

9 rue du Pâquis, 55260 Raival (France)

FSchwindt@ac-nancy-metz.fr 
